\title{
LETTERS
}

\section{T cell recognition of a non-protein antigen preparation of Campylobacter jejuni in patients with Guillain-Barré syndrome}

\author{
J C Cooper, S Hughes, A Ben-Smith, C O S Savage, J B Winer
}

J Neurol Neurosurg Psychiatry 2002;72:413-417

$\mathrm{E}$ vidence is accumulating that anti-ganglioside antibodies may well mediate both Miller Fisher syndrome and the acute motor axonal form of Guillain-Barré syndrome ${ }^{1}$ but such antibodies are not present in most patients with the more common demyelinating forms of Guillain-Barré syndrome. T cells are thought to play an important part in the pathogenesis of the neuropathy in these patients, by analogy with experimental allergic neuritis and from histological studies of biopsy and postmortem peripheral nerve material. Furthermore many anti-ganglioside antibodies are of the IgG isotype normally requiring $\mathrm{T}$ cell help in their production.

We have previously proposed that $\gamma \delta \mathrm{T}$ cells might have a role in the pathogenesis of inflammatory demyelinating neuropathy after Campylobacter jejuni infection of the gut, because of their potential to react with carbohydrate ligands. ${ }^{2}$ It is possible to culture $\gamma \delta \mathrm{T}$ cell lines from peripheral nerve biopsy material from patients with demyelinating forms of GuillainBarré syndrome ${ }^{2}$ and demonstrate immunostained $\gamma \delta$ T cells in nerve infiltrates. ${ }^{3} \mathrm{CDl}$ is an essential presentational molecule in the interaction of $\gamma \delta \mathrm{T}$ cells and their ligands and we and others have shown that CDI is upregulated in biopsy material from both Guillain-Barré syndrome and chronic inflammatory demyelinating polyneuropathy. ${ }^{34}$

To investigate the hypothesis that $\gamma \delta$ T cells from patients with Guillain-Barré syndrome might respond to non-protein antigens, we carried out a 6 day proliferation assay using peripheral blood mononuclear cells (PBMCs) from 23 patients with Guillain-Barré syndrome using techniques similar to those previously reported. ${ }^{5}$ Nine of these patients were known to have serological evidence of preceding $C$ jejuni infection. An isolate of $C$ jejuni cultured from a patient with Guillain-Barré syndrome (Penner type 04) was used at a concentration of 5 $\mu \mathrm{g} / \mathrm{ml}$ and treated with a standard protocol of proteinases to abrogate T cell responses to peptides. ${ }^{6}$ The effectiveness of this protocol in removing peptides was assessed by overloading an SDS-PAGE gel with this antigen and confirming that no protein remained in the preparation. Patients with multiple sclerosis, other neurological diseases, and healthy controls were studied for comparison. Proliferative responses among these groups are shown in figure 1. Proliferative responses greater than normal controls or other neurological diseases (stimulation index $\geqslant 10$ ) were seen in two patients with Guillain-Barré syndrome and in two patients with multiple sclerosis. In one patient with Guillain-Barré syndrome the stimulation index was $>52$ times the background level.

Ethical permission was obtained to peform sural nerve biopsies in eight patients with Guillain-Barré syndrome after $C$ jejuni infection and expression of CDlb by immunohistochemistry was upregulated in seven, confirming similar findings to those found in two patients studied by Khalili-Shirazi et al. ${ }^{3}$

Our results suggest that a proportion of patients with demyelinating disorders show evidence of proliferative responses to non-protein antigens. Our results are still preliminary and we propose further work to determine the exact nature of the

Abbreviations: PBMCs, peripheral blood mononuclear cells

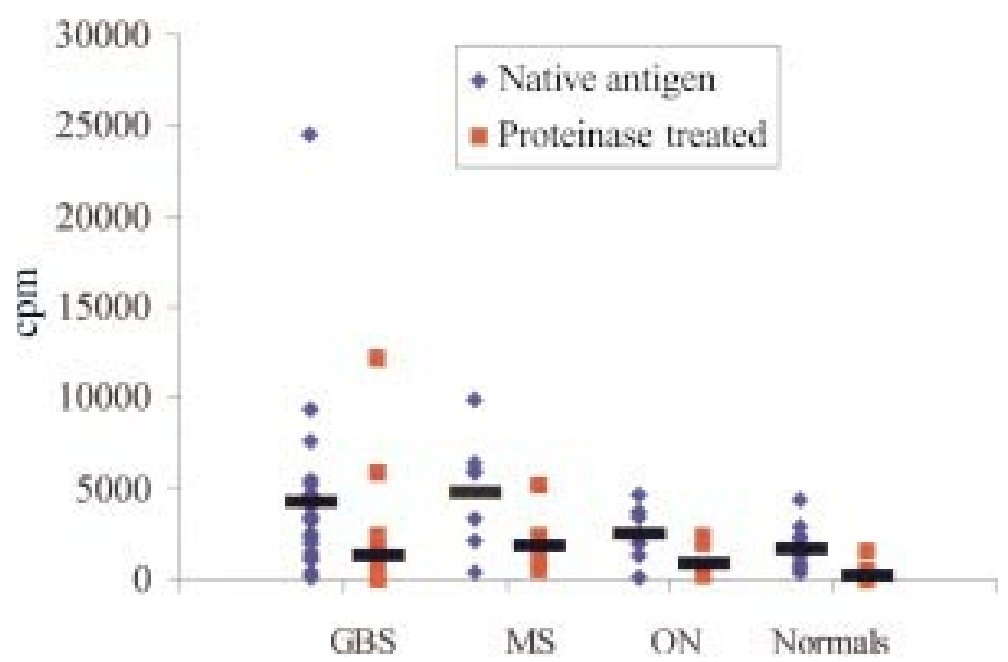

Figure 1 Proliferation to $C$ jejuni antigens. Proliferation (mean of triplicates-background count for cells alone) of PBMCs isolated from patients with Guillain-Barré syndrome (GBS), multiple sclerosis (MS), other neurological disorders (ON), and normal healthy volunteers (Normals). Proliferations are subdivided into untreated $C$ jejuni and protease resistant $C$ jejuni. The mean stimulation value for each patient group is represented by the horizontal bar. 
responsible antigen so that we can compare our results with serological detection of specific anti-ganglioside antibodies. The results are remarkable as it is known that very many different carbohydrate antigenic determinants are present in different strains of $C$ jejuni and our antigen was obtained from a single isolate, providing an extremely limited array of possible antigens. Whereas we anticipated such responses in patients with Guillain-Barré syndrome the positive results in patients with multiple sclerosis are also of interest. These results would accord with studies implicating immunity to non-protein antigens in multiple sclerosis, including a study in which it was possible to generate ganglioside specific clones from patients with multiple sclerosis that were restricted by CDIb. ${ }^{7}$

Further study of non-classic $\mathrm{T}$ cell responses in both Guillain-Barré syndrome and multiple sclerosis may prove informative.

\section{ACKNOWLEDGEMENTS}

We acknowledge support for our ongoing research from both The Wellcome Trust and the Guillain-Barré Syndrome Support Group and are grateful to Dr Cheeseborough for advice about the $C$ jejuni serology.

\section{Authors' affiliations}

J C Cooper, A Ben-Smith, C O S Savage, Birmingham Centre for Immune Regulation, Division of Medical Sciences, The Medical School, University of Birmingham, Edgbaston, Birmingham, UK
S Hughes, J B Winer, Department of Neurosciences, Queen Elizabeth Hospital, Edgbaston, Birmingham B15 2TH, UK

Correspondence to: Dr J B Winer; j.b.winer@bham.ac.uk

Received 3 August 2000

In final revised form 9 October 2001

Accepted 23 October 2001

\section{REFERENCES}

1 Sheikh KA, Griffen JW. Variants of the Guillain-Barré syndrome: progress toward fulfilling "Koch's postulates". Ann Neurol 2001;49:694-5

2 Ben-Smith, A, Gaston JS, Barber PC, et al. Isolation and characterisation of $T$ lymphocytes from sural nerve biopsies in patients with Guillain-Barré syndrome and chronic inflammatory demyelinating polyneuropathy. J Neurol Neurosurg Psychiatry 1996;61:362-8.

3 Khalili-Shirazi AGN, Londei M, Summers L, et al. The distribution of CD1 molecules in inflammatory neuropathy. J Neurosci 1998;158:154-63.

4 Winer JB, Hughes S, Cooper J, et al. T cells infiltrating nerve biopsies from patients with inflammatory neuropathy. J Neurol (in press).

5 Ben-Smith A, Goodall JC, Gaston JS, et al. Stimulation of peripheral blood lymphocytes with Campylobacter jejuni generates a $\gamma \delta$ T cell response in patients with Guillain-Barre syndrome. Clin Exp Immunol 1997; 109:121-6.

6 Pfeffer K, SB, Gulle H, et al. Primary responses of human T cells to mycobacteria: a frequent set of $\gamma \delta \mathrm{T}$ cells are stimulated by protease resistant ligands. Eur J Immunol 1990;20: 1175-9.

7 Shamshiev A, Donda A, Caren I, et al. Self glycolids as T cell autoantigens. Eur J Immunol 1999;29:1667-75.

\title{
Changes in excitability of motor cortex in severe hepatic failure
}

\author{
A Oliviero, R Gaspari, P A Tonali, M A Pennisi, G Mercurio, F Pilato, R Proietti, \\ V Di Lazzaro
}

J Neurol Neurosurg Psychiatry 2002;72:414-416

S evere liver failure causes accumulation of both albumin bound and water soluble substances that exhibit toxic effects on the brain and lead to hepatic encephalopathy. This is a complex neuropsychiatric syndrome determined by a neurotransmission failure rather than from primary energy deficit. ${ }^{1}$ Transcranial magnetic stimulation represents a noninvasive method that can be used to study the physiology of the human brain. ${ }^{2}$ Commonly used transcranial magnetic stimulation parameters include motor thresholds, cortical silent period (CSP), central motor conduction time (CMCT), intracortical inhibition (ICI), and intracortical facilitation. The threshold for producing motor evoked potentials reflects the excitability of a central core of neurons, which arises from the membrane excitability and a balance between inhibitory and excitatory input from local circuits. The CMCT provides information on the corticospinal tract anatomical integrity, CSP and ICI reflect the activity of GABAergic inhibitory circuits. ${ }^{2-6}$ Additional in vivo information on central motor circuits can be obtained using transcranial electrical stimulation of the motor cortex. transcranial electrical stimulation stimulates the motor areas of the human brain deeply in the white matter and is therefore insensitive to changes in cortical excitability. ${ }^{6}$ Combining the data from transcranial electrical stimulation and transcranial magnetic stimulation it is possible to determine the site, cortical or subcortical, where brain functional changes have taken place. We used transcranial magnetic stimulation to explore function of the cerebral cortex in five patients affected by severe liver failure. Recently, a molecular adsorbent recirculating system (MARS) has been proposed as a new extracorporeal detoxifying treatment. ${ }^{7}$ We used transcranial magnetic stimulation to evaluate the effects of MARS treatment on functions of the cerebral cortex in these patients and also the effects of orthotopic liver transplantation in one of them.

Clinical and neurophysiological examinations were recorded at baseline and 12-18 hours after the MARS procedure, combined with standard haemodialysis (Integra-Hospal) in patient 1, 3, 4, and 5 and with continous venovenosus haemodialyis (CVVHD) (BAXTER System BMIl-BMl4) in patient 2. Neurophysiological findings from the patients were compared with the data from 10 healthy age matched control subjects (mean age 35 (SD 17) years). Patients' relatives gave informed consent to participate in the study, which was approved by the local ethics committee. Patient 1, a 28 year old white woman developed a delayed graft dysfunction 3 days after orthoptic liver transplantation for a Budd-Chiari syndrome. On admission to the intensive care unit, her simplified acute physiology score II (SAPS II) ${ }^{8}$ ) was 67 and she was in a deep coma.

Abbreviations: CSP, cortical silent period; CMCT, central motor conduction time; $\mathrm{ICl}$, intracortical inhibition; MARS, molecular adsorbent recirculating system; CVVHD, continous venovenosus haemodialyis; SAPS, simplified acute physiology score II; RMT, resting motor threshold; AMT, active motor threshold; ISI, interstimulus interval 


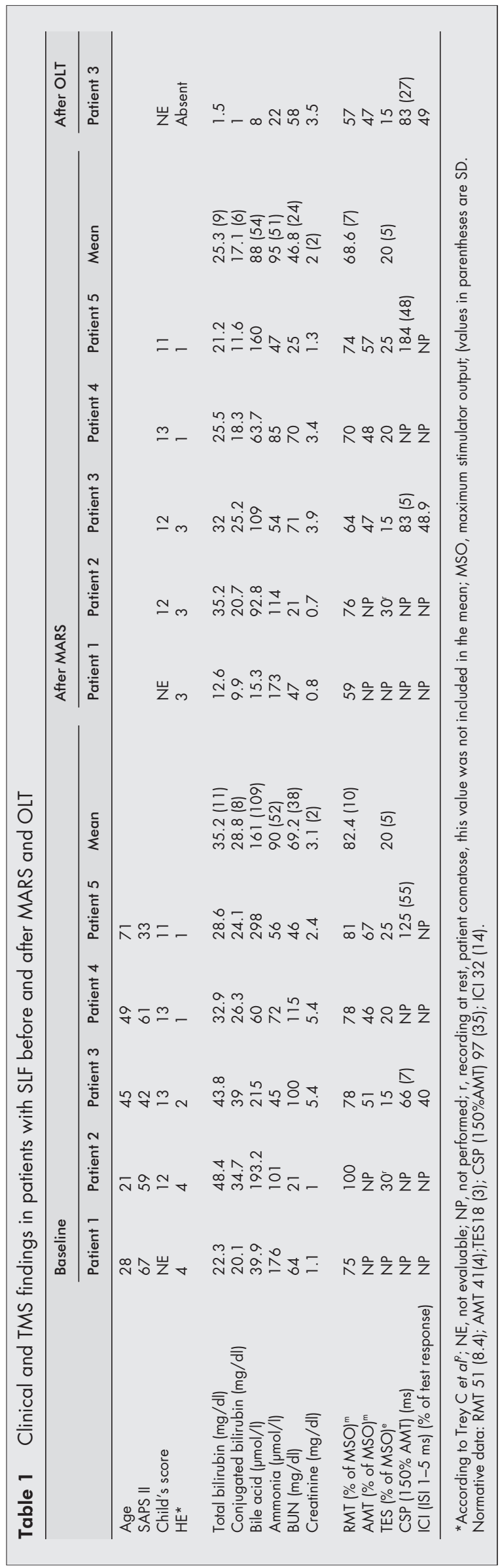

She underwent a MARS procedure with standard haemodialysis and after a single treatment we documented the improvement of hepatic encephalopathy. She was drowsy but responsive to verbal stimuli. The patient was discharged from our intensive care unit and died because of cardiac tamponade 3 months later.

Patient 2 was a 21 year old non-white woman affected by Wilson's disease and listed for orthotopic liver transplantation. On admission to the intensive care unit, her SAPS II was 59. The patient was in a deep coma and MARS treatment with CVVHDF was started. After a single MARS session, we documented a mild recovery of motor activity and gag reflexes. The patient died because of severe respiratory failure.

Patient 3 was a 45 year old white man affected by HBV related cirrhosis, listed for or thotopic liver transplantation. On admission to the intensive care unit, his SAPS II was 42 . He was drowsy and responsive to verbal stimuli. Six MARS treatments with standard haemodialysis were performed. After each session, we noted neurological status improvement. On the 36th day from admission he was successfully transplanted. We report transcranial magnetic stimulation data recorded before and after the first of the six MARS treatments and 1 month after orthotopic liver transplantation.

Patient 4 was a 49 year old white man affected by alcoholic cirrhosis. On admission to the intensive care unit, his SAPS II was 61 . He was drowsy and responsive to verbal stimuli. He underwent a MARS procedure with standard haemodialysis and after a single treatment the clinical picture was not modified. The patient died because of multiple organ failure.

Patient 5 was a 71 year old white man with subacute hepatic failure due to hepatotoxicity induced by antituberculosis treatment (isoniazide and rifampicine). On admission to the intensive care unit, his SAPS II was 33. He was drowsy and responsive to verbal stimuli. He underwent the MARS procedure with standard haemodialysis and after a single treatment the clinical picture was only slightly modified.

Magnetic stimulation was performed with a high power Magstim 200 (Magstim Co, Whitland, Dyfed). A figure of eight coil with external loop diameters of $9 \mathrm{~cm}$ was held over the right motor cortex at the optimum scalp position to elicit motor responses in the contralateral first dorsalis interosseus.

Resting motor threshold (RMT) and active motor threshold (AMT) were defined as the minimum stimulus intensity that produced a liminal motor evoked response (about $50 \mu \mathrm{V}$ in $50 \%$ of trials) at rest and during isometric contraction of the tested muscle at about $20 \%$ maximum respectively. The AMT was evaluated only in patients 3,4 , and 5 because patients 1 and 2 were comatose. The CMCT was calculated by subtracting the peripheral conduction time from spinal cord to muscles from the latency of responses evoked by cortical stimulation. Silent period duration was evaluated only in patients 3 and 5 . The silent period was elicited while the patients held a tonic voluntary contraction of about $50 \%$ of maximum voluntary contraction. Five stimuli at $150 \%$ AMT were given. Paired pulse stimulation to evaluate the intracortical inhibition was performed only in patient 3. Intracortical inhibition was studied using the technique of Kujirai et al. ${ }^{35}$ Interstimulus intervals (ISIs) between 1 and 5 ms were investigated. Five stimuli were delivered at each ISI. Amplitude of the conditioned EMG responses was expressed as percentage of the amplitude of the test EMG responses. The amplitude of the conditioned responses at the five different ISIs studied was averaged, obtaining a grand mean amplitude. In patients 2, 3 , 4 , and 5 we have also analysed the motor threshold of electrical anodal stimulation. Electrical stimulation was performed with a Digitimer D180A stimulator, with a $50 \mu$ s time constant. The cathode was located at the vertex and the anode $7 \mathrm{~cm}$ laterally (anodal stimulation). The stimulation was performed at rest in patient 2 and during voluntary contraction in patients 3, 4, and 5. Electrophysiological parameters were re-evaluated in the patients after extracorporeal treatment. 
An unpaired $t$ test was used to compare patient results with the data of 10 healthy age matched control subjects (mean age 35 (SD 17) years). Analysis of variance (ANOVA) for repeated measures was used to analyse the MARS effects on RMT, transcranial electrical stimulation, and AMT. If the effect of a given parameter was significant, data were further compared post hoc using Student's $t$ test for paired samples.

Electrophysiological results are summarised in table 1. The RMT was significantly higher in the patients studied than in the control group (82.4 (SD 10)\% v 51 (SD 8.4)\%; p<0.01) and decreased significantly after MARS (68.6 (SD 7)\% $v 82.4$ (SD $10) \%, p=0.010)$. The AMT was higher in the three patients studied than in the control group (54.7 (SD 11)\% v 41 (SD 4)\%, $\mathrm{p}>0.05$ ) but the difference was not statistically significant probably because of the restricted number. In the patients the AMT was slightly decreased after the MARS procedure (50.7 (SD 5.5)\% $v 54.7$ (SD 11)\%, p>0.05, paired $t$ test). There was no difference between the CMCT and CSP and ICI of the studied patients and of controls. Active motor threshold to transcranial electrical stimulation was similar in the three studied patients and in the control group (20 (SD 5)\% $v 18$ (SD $3) \%, p>0.05)$. In patient 3 we found normalisation of all the parameters evaluated after orthotopic liver transplantation.

We demonstrated hypoexcitability of the motor cortex (increased RMT) in patients with severe liver failure. As transcranial electrical stimulation was normal, this hypoexcitability originates at the cortical level. ${ }^{2}{ }^{6}$ Cortical hypoexcitability found using transcranial magnetic stimulation could be explained by decreased glutamatergic excitatory neurotransmission and/or increased GABAergic inhibitory neurotransmission, previously reported in severe liver failure. ${ }^{1}$ Transcranial magnetic stimulation study led us to interpret the hypoexcitability of the motor cortex in severe liver failure as resulting from underactivity of cortical glutamatergic excitatory circuits rather than increased GABAergic activity (normal CSP and ICI). Electrophysiological abnormalities in our patients were reversed by MARS treatment or by orthotopic liver transplantation. However, the few patients presented does not allow us to express any consideration about the clinical implication of MARS treatment on the outcome of patients with severe liver failure.
These preliminary findings suggest that transcranial magnetic stimulation may be a useful tool in assessing patients with severe liver failure.

\section{ACKNOWLEDGEMENTS}

We thank A Accurso for technical help. Competing interests: GM has been reimbursed by Integra-Hospal for attending a conference.

\section{Authors' affiliations}

A Oliviero, P A Tonali, F Pilato, V Di Lazzaro, Institute of Neurology, Catholic University, Largo A Gemelli 8, 00168 Rome, Italy R Gaspari, M A Pennisi, G Mercurio, R Proietti, Institute of Anesthesiology, Catholic University, Rome, Italy

P A Tonali , IRCCS "Casa Sollievo della Sofferenza", San Giovanni Rotondo, Italy

Correspondence to: Dr V Di Lazzaro, Istituto Di Lazzaro, Università Cattolica, Largo A Gemelli 8, 00168 Rome, Italy;

vdilazzaro@rm.unicatt.it

Received 7 September 200

In revised form 12 November 2001

Accepted 14 November 2001

\section{REFERENCES}

1 Hazell AS, Butterworth RF. Hepatic encephalopathy: an update of pathophysiologic mechanisms. Proc Soc Exp Biol Med

1999;222:99-112.

2 Hallet $M$. Transcranial magnetic stimulation and the human brain Nature 2000;406: 147-50.

3 Kujirai T, Caramia MD, Rothwell JC, et al. Corticocortical inhibition in human motor cortex. J Physiol (Lond) 1993;471:501-19.

4 Ziemann U, Lonnecker S, Steinhoff BJ, et al. Effects of antiepileptic drugs on motor cortex excitability in humans: a transcranial magnetic stimulation study. Ann Neurol 1996:40:367-78.

5 Di Lazzaro V, Oliviero A, Meglio M, et al. Direct demonstration of the effect of lorazepam on the excitability of the human motor cortex. Clin Neurophysiol 2000;1 11:794-9.

6 Di Lazzaro V, Oliviero A, Profice P, et al. Comparison of descending volleys evoked by transcranial magnetic and electric stimulation in conscious humans. Electroencephalogr Clin Neurophysiol 1998;109:397-401.

7 Mitzner SR, Stange J, Klammt S, et al. Improvement of hepatorenal syndrome with extracorporeal albumin dialysis MARS: results of a prospective, randomized, controlled clinical trial. Liver Transpl 2000;6:277-86.

8 Le Gall JR, Lemeshow S, Saulnier F. A new simplified acute physiology score (SAPS II) based on a European/North American multicenter study. JAMA 1993:270:2957-63.

9 Trey C, Burns DG, Saunders SJ. Treatment of hepatic coma by exchange blood transfusion. N Engl J Med 1966;274:473-81.

\section{Amnesia for childhood in patients with unexplained neurological symptoms}

\section{J Stone, M Sharpe}

n $\mathrm{n}$ a preliminary study, we tested the hypothesis that patients with medically unexplained symptoms attending the clinic of a general adult neurologist would have delayed earliest and continuous memories compared with patients whose symptoms were explained by neurological disease. Depression, adverse childhood experience, and low socioeconomic status have all been associated both with poor memory of childhood. Because these variables are also associated with medically unexplained symptoms we hypothesised that we would find a link between unexplained symptoms and impaired memories of childhood.

One hundred consecutive neurology outpatients were asked the question "What is the very first thing that you can remember?" and "How old were you at the time?". They were then asked "From what age from could you produce your own life story, biography, or CV without help from a parent or relative?" and "Do you have blanks in your memory?". Neurological diagnoses were recorded and the patient completed the brief assessment scale for depression cards (BASDEC) scale for depression. ${ }^{1}$ This simple self rated measure is scored out of 21 . A score of 7 or more is taken as the threshold for depression.

Fifty five patients were diagnosed with neurological disease and 45 were diagnosed with unexplained neurological symptoms by a consultant neurologist (GE). The neurological

Abbreviations: BASDEC, brief assessment scale for depression cards 
Table 1 Results in 100 consecutive neurology outpatients

\begin{tabular}{|c|c|c|c|}
\hline & $\begin{array}{l}\text { Neurological } \\
\text { disease } \\
(n=55)\end{array}$ & $\begin{array}{l}\text { Unexplained } \\
\text { neurological } \\
\text { symptoms }(n=45)\end{array}$ & Significance \\
\hline Age (median) & 44 & 47 & $\begin{array}{l}\text { NS } \\
\text { Unpaired } t \text { test }\end{array}$ \\
\hline Sex & $28 \mathrm{~F}: 27 \mathrm{M}$ & $36 \mathrm{~F}: 9 \mathrm{M}$ & $\begin{array}{l}p<0.005 \\
\text { Fisher's exact test }\end{array}$ \\
\hline Reported age of earliest memory (y) (median) & 4 & 5 & $\begin{array}{l}\text { NS } \\
\text { Mann-Whitney }\end{array}$ \\
\hline Reported age of onset of continuous memory (y) (median) & 6 & 11 & $\begin{array}{l}p<0.005 \\
\text { Mann-Whitney }\end{array}$ \\
\hline "Memory blanks?" (number of true responses) & 3 & 17 & $\begin{array}{l}p<0.0001 \\
\text { Fisher's exact test }\end{array}$ \\
\hline Number with BASDEC score $\geqslant 7$ (threshold for depression) & 13 & 23 & $\begin{array}{l}p<0.01 \\
\text { Fisher's exact test }\end{array}$ \\
\hline
\end{tabular}

diagnoses were epilepsy (12), multiple sclerosis (10), migraine (13), Parkinson's disease (four), cervical spondylosis (three), brain space occupying lesion (three), transient ischaemic attack (two), syncope (two), and one each of motor neuron disease, torticollis, benign positional vertigo, cerebellar degeneration, neuropathy, and drug toxicity. The presenting unexplained neurological symptoms were headache (18), dizziness/balance problems (eight), sensory symptoms (seven), pain (six), memory complaints (three), diplopia (one), weakness (one), and non-epileptic attacks (one).

The results are shown in table 1 . There was no significant difference between the two groups when asked about their earliest memory although both groups reported ages that were somewhat later than the average age suggested in the literature. Patients with unexplained symptoms had significantly later onset of reported continuous memory $(p<0.005)$ and reported more memory blanks $(p<0.001)$. There were significantly more women in the unexplained group but the age differences were not significant.

Depression was more common in the unexplained group $(51 \%)$ versus the explained group (24\%), as might be expected $(p<0.05)$. Therefore could the effect on memory seen in the unexplained group be entirely due to depression? Although depression and unexplained symptoms overlap considerably, using a general linear model they were both independently related to continuous memory $(\mathrm{p}<0.05)$. Neither the sex nor the age of the patients were significant in this model.

Research on earliest memories has placed their onset at between 3-4 years in healthy adults. ${ }^{2}$ This age is thought to be increased by a wide range of variables including male sex, depression, adverse childhood experience, low verbal IQ, low socioeconomic status, ethnic factors, parental retelling of events, and the presence of siblings. ${ }^{3}$ Similar factors have been shown to influence autobiographical memory. In this study, although depression could be the determining factor, socioeconomic status, and IQ could plausibly have been unequally distributed in the two groups as well. The methods in this study were also relatively crude and unblinded. In particular, we did not attempt to validate the patient's reports using contextual cues or documented events. Both of these strategies could have reduced differences between the groups. The questions we asked, however, which were really a measure of perception of childhood memories, at least have the advantage of reflecting everyday neurological practice.

If depression and adverse childhood experience are key factors in determining early memories in patients with unexplained symptoms, what are the possible mechanisms? Some studies have suggested that patients with depression tend to have "overgeneral" recall of memories-that is, recall of general events rather than detail of positive or negative events. ${ }^{4}$ This may bring forward the age they think that they can confidently remember their own history. Similar effects have been found in borderline personality disorder, posttraumatic stress disorder, and in some but not all adults who report abuse as a child. ${ }^{5}$ It has been suggested in these contexts that dissociation and repression may be mechanisms that help people avoid unpleasant memories. ${ }^{5}$ Alternatively, what we have found may simply reflect a perception of amnesia for childhood in patients with unexplained neurological symptoms rather than actual amnesia. Regardless of the mechanisms, associations between illness states and poor reported childhood memories may have clinical relevance. Inability to adequately remember positive events from childhood may be a maintaining factor in depression and thus a target for treatment. The same may be true in many patients with unexplained neurological symptoms.

This study suggests a difference in age of onset of reported autobiographical memory but not the age of reported earliest memory in patients with unexplained neurological symptoms. Further studies using standardised measures of recall may help to disentangle the relation between unexplained neurological symptoms, memory complaints, emotional disorder, and adverse childhood experience.

\section{Authors' affiliations}

J Stone, Department of Clinical Neurosciences, Western General Hospital, Crewe Rd, Edinburgh EH4 2XU, UK

M Sharpe, Department of Psychiatry, Royal Edinburgh Hospital,

Morningside Park, Edinburgh, UK

G Elrington, Colchester General Hospital, Turner Road, Colchester CO4 5JL, UK

Correspondence to: Dr J Stone, Department of Clinical Neurosciences, Western General Hospital, Crewe Rd, Edinburgh EH4 2XU, UK; jstone@skull.dcn.ed.ac.uk

Received 21 June 2001

In revised form 29 October 2001

Accepted 7 November 2001

\section{REFERENCES}

1 Adshead F, Cody DD, Pitt B. BASDEC: a novel screening instrument for depression in elderly medical inpatients. BMV 1992:305:397.

2 Pillemer DB, White SH. Childhood events recalled by children and adults. Adv Child Dev Behav 1989;21:297-340.

3 Bruhn AR. Early memories and maladjustment: comment on Spirrison, et al. and recalled age of earliest memory. Psychol Rep 1998;82:128792 .

4 Brittlebank AD, Scott J, Williams JM, et al. Autobiographical memory in depression: state or trait marker? Br J Psychiatry 1993;162:1 18-21.

5 Jones B, Heard $H$, Startup $M$, et al. Autobiographical memory and dissociation in borderline personality disorder. Psychol Med 1999;29:1397-404. 